\title{
Tratamiento psicológico de mujeres adultas víctimas de abuso sexual en la infancia: resultados a largo plazo
}

\author{
Belén Sarasua ${ }^{1}$, Irene Zubizarreta ${ }^{1},{\text { Paz de } \text { Corral }^{2} \text { y Enrique Echeburúa }}^{2 *}$ \\ ${ }^{1}$ Centro de Tratamiento Psicológico para Victimas de Violencia de Género de la Diputación Foral de Álava \\ ${ }^{2}$ Universidad del País V asco UPV/EHU. Cibersam
}

\begin{abstract}
Resumen: El abuso sexual (ASI) constituye la victimización más frecuente en la infancia. Los efectos del ASI en la vida adulta pueden afectar al funcionamiento, físico, psicológico y social y generar alteraciones psicopatológicas. El objetivo de este estudio fue evaluar un programa individual cognitivo-conductual, de 12 sesiones, en el tratamiento de mujeres adultas víctimas del ASI, llevado a cabo durante 20 años en un marco comunitario. La muestra constó finalmente de 121 sujetos que participaron voluntariamente en el estudio. Se utilizó un diseño de medidas repetidas con seguimientos de 1, 3, 6 y 12 meses. La tasa de éxitos en el tratamiento del trastorno de estrés postraumático fue del $90.7 \%$ en las víctimas que completaron el tratamiento. A pesar de la pérdida de pacientes en los seguimientos $(25.58 \%)$, los resultados se mantuvieron en las pacientes evaluadas en los cuatro periodos establecidos (un $74.42 \%$ al cabo de 12 meses), con una alta reducción de las recaídas. Sin embargo, hubo un nivel más bien alto de rechazos y de abandonos. Una conclusión es que hay que desarrollar estrategias motivacionales para mantener a las víctimas en el tratamiento. Se comentan las implicaciones de este estudio para la práctica clínica y para la investigación. Palabras clave: Abuso sexual infantil; mujeres víctimas adultas del ASI; tratamiento psicológico; seguimiento a largo plazo.
\end{abstract}

\section{Introducción}

El abuso sexual infantil (ASI) es un fenómeno frecuente que, sin embargo, se ha mantenido tradicionalmente oculto. Según un reciente meta-análisis de Pereda, Guilera, Forns y Gómez-Benito (2009), la tasa de prevalencia de algún tipo de abuso sexual en menores en la población general de diversos países es de un 7.4\% en el caso de los niños y de un $19.2 \%$ en el de las niñas. Si bien el abuso sexual grave, con contacto físico, con carácter repetido y con una interferencia claramente negativa en el desarrollo emocional del menor, es bastante más reducido (Echeburúa y Guerricaechevarría, 2011), estas cifras dan idea de la gravedad de este hecho.

Los efectos de la victimización a largo plazo son, en general, muy negativos para el funcionamiento psicológico de las víctimas, sobre todo cuando el agresor ha sido un miembro de la misma familia y cuando se ha producido un abuso sexual con penetración. Si bien estas consecuencias de largo alcance son variables de unos casos a otros, hay una estrecha relación entre el ASI y la aparición de alteraciones emocionales en la juventud y en la vida adulta. Así, las víctimas adultas de ASI pueden experimentar un trastorno de estrés postraumático crónico, una sintomatología ansioso-depresiva, un comportamiento sexual insatisfactorio, trastornos de personalidad (especialmente el trastorno antisocial y el trastorno límite), alteraciones de la conducta

* Dirección para correspondencia [Correspondence address]:

Enrique Echeburúa. Facultad de Psicología. Avda. de Tolosa, 70. 20018

San Sebastián (España).E-mail: enrique.echeburua@ehu.es
Title: Psychological treatment of adult female survivors of childhood sexual abuse: long term results.

Abstract: Child sexual abuse (CSA) is one the most common forms of child victimization. The effects of CSA can extend into adulthood, impacting on physical, psychological, and social functioning and contributing to adult psychopathology. The aim of this paper was to evaluate an individual 12 sessions cognitive-behavioral programme in the treatment of female adult survivors of CSA after implementing it for twenty years in a community setting. The sample consisted of 121 subjects who voluntarily participated in the study. A design with repeated measures (pre-treatment, posttreatment and 1-, 3-, 6- and 12-month follow-up) was used. The success rate regarding posttraumatic stress disorder was of $90.7 \%$ in the treatment completers. In spite of the lost patients in the follow-ups $(25.58 \%)$, the results were maintained in the contacted patients at follow-up $(74.42 \%$ in the 12-month-follow-up), with a high impact on reducing recidivism. However, the rate of refusals and drop-outs of the treatment was rather high. Therefore it is necessary to develop strategies to improve motivation for treatment. Implications of this study for clinical practice and future research in this field are commented upon.

Key words: Child sexual abuse; female adult survivors of CSA; psychological treatment; long-term outcome.

alimentaria, abuso de alcohol o drogas o una adaptación inadecuada a la vida cotidiana (Fergusson, Boden y Horwood, 2008; Flitter, Elhai y Gold, 2003; Kendler, Bulik, Silberg, Hettema, Myers y Prescott, 2000; McLean y Gallop, 2003; Pereda, Gallardo-Pujol y Jiménez Padilla, 2011; Putnam, 2003). En otros casos pueden aparecer alteraciones psicosomáticas en la vida adulta (por ejemplo, problemas respiratorios o gastrointestinales, sobrepeso, dolores de cabeza persistentes, consultas frecuentes a los Centros de Salud, etcétera) como resultado directo o indirecto de un abuso sexual continuado en la infancia (James, 2008; Maniglio, 2009). En conjunto, las víctimas de ASI pueden experimentar en la vida adulta una tasa de trastornos mentales 2.4 veces mayor que las personas no expuestas al ASI (Fergusson et al., 2008).

El impacto psicológico del ASI va a depender de la frecuencia, de la duración, de la gravedad del abuso y de la relación con el agresor, pero también de la fase del desarrollo psicológico de la víctima en la que tuvo lugar el suceso (Briere y Elliott, 2003; Katz y Watkins, 1998). A su vez, el papel de los factores amortiguadores -tales como las relaciones familiares cohesionadas, la red de apoyo social o la autoestima positiva- en la reducción del impacto psicológico parece sumamente importante, pero está aún por esclarecer (Cortés, Cantón-Cortés y Cantón, 2011; Echeburúa, Corral y Amor, 2002; Patró, Corbalán y Limiñana, 2008).

En general, hay una documentación abundante sobre la epidemiología del abuso sexual en menores (Pereda et al., 2009), sobre las repercusiones psicológicas de este suceso 
traumático en la estabilidad emocional de las víctimas (Cortés et al., 2011) e incluso sobre la credibilidad del testimonio (Echeburúa y Subijana, 2008), pero, sin embargo, todavía resulta insuficiente la bibliografía existente sobre los aspectos clínicos de la intervención psicológica (Taylor y Harvey, 2010).

Ha habido algunos tratamientos que han mostrado ser útiles para hacer frente al trastorno de estrés postraumático en víctimas adultas de abuso sexual en la infancia. Entre ellos se han incluido la terapia de exposición, la reestructuración cognitiva, el entrenamiento en inoculación de estrés y la terapia de procesamiento cognitivo. Así, por ejemplo, Smucker, Dancu, Foa y Niederee (1995) han propuesto una "reescritura" de imágenes, apoyada en la exposición en imaginación y la reestructuración cognitiva, en donde se trata de sustituir las imágenes de victimización por imágenes de control en un programa de nueve sesiones. La propuesta de Resnick y Newton (1992) se ha basado en una combinación de la terapia de exposición con el entrenamiento en inoculación de estrés y ha incluido, entre otras, técnicas tales como la respiración controlada, la relajación muscular o las autoinstrucciones. A su vez, Jehu (1989) mostró que la reestructuración cognitiva era útil para desmontar las distorsiones cognitivas de las víctimas y para mejorar su estado de ánimo. Se trata, sin embargo, de estudios no controlados.

Si bien se cuenta en general con terapias empíricamente validadas para el trastorno de estrés postraumático (Foa, Keane y Friedman, 2003), el apoyo empírico a los tratamientos psicológicos para el ASI es aún escaso (Taylor y Harvey, 2010). Los estudios controlados en esta área, con muestras bien definidas, grupos de control adecuados y un seguimiento a largo plazo, son casi inexistentes. Además, lo que las víctimas adultas de abuso sexual infantil comparten es un suceso traumático común, pero no un trastorno específico (Maniglio, 2009). De hecho, al ser los efectos del ASI a largo plazo variados e inespecíficos, las propuestas terapéuticas han sido asimismo muy diversas. Si bien la terapia psicológica para las víctimas adultas del ASI parece beneficiosa para mejorar el malestar emocional, hasta la fecha ningún tratamiento ha mostrado una clara superioridad ni tampoco ningún formato (individual o grupal) o duración específicos (breve o prolongado) han probado ser superiores a otros (Martsolf y Draucker, 2005; Taylor y Harvey, 2010).

El carácter poco concluyente de los resultados obtenidos hasta la fecha hace aconsejable la presentación de nuevos estudios. Por ello, el objetivo de este artículo es presentar los resultados sistemáticos obtenidos con un tratamiento cognitivo-conductual aplicado a una muestra amplia de mujeres adultas víctimas de abuso sexual en la infancia, con un seguimiento largo ( 1 año), y centrado en diversos objetivos terapéuticos (trastorno de estrés postraumático, malestar emocional y evitación de contactos sexuales deseados).

\section{Método}

\section{Participantes}

La muestra total de este estudio consta de 131 víctimas adultas que han sufrido abusos sexuales en la infancia y que han acudido en busca de un tratamiento psicológico al Centro de Asistencia Psicológica para Víctimas de Violencia de Género de la Diputación Foral de Álava (País Vasco). En dicho centro se desarrolla un programa de tratamiento específico para víctimas de agresiones sexuales desde 1990 y la atención es gratuita para las pacientes.

Los criterios de selección han sido los siguientes: a) ser mujer mayor de 18 años y haber sido víctima de abusos sexuales en la infancia; b) no sufrir actualmente un proceso de victimización ni un trastorno mental grave; y c) acceder voluntariamente a la participación en el estudio tras haber sido ampliamente informada, con la firma de una hoja de consentimiento.

La muestra preseleccionada abarcó a las 150 mujeres que habían acudido al programa. Sin embargo, 7 (el 4.7\%) fueron excluidas por sufrir violencia por parte de la pareja y 12 (el $8 \%$ ) por padecer un trastorno mental grave (trastorno bipolar y límite fundamentalmente). La muestra seleccionada finalmente fue, por tanto, de 131 mujeres.

Respecto a las características demográficas, las víctimas eran mujeres jóvenes, con una edad media de 27.8 años ( $D T=9.2$; rango: $18-54)$, no tenían pareja en una mayoría de los casos $(n=80$; el $61.1 \%)$, eran de nacionalidad española $(n=118 ; 90.1 \%)$ y pertenecían a un nivel socioeconómico medio $(n=67 ; 51.1 \%)$ o bajo $(n=56 ; 42.7 \%)$.

\section{Diseño}

Es un estudio unicéntrico en el que se ha utilizado un diseño de grupo único de medidas repetidas antes-después. La evaluación de todas las participantes se ha efectuado en el pretratamiento, en el postratamiento y en los seguimientos de 1, 3, 6 y 12 meses.

En esta investigación no se ha recurrido a un grupo de control sin tratamiento o de lista de espera porque en las víctimas adultas de abuso sexual en la infancia los síntomas tienden a cronificarse y la probabilidad de una recuperación espontánea es escasa (Kilpatrick y Cahoun, 1988; Echeburúa, Corral, Zubizarreta y Sarasua, 1995, 1997) y porque se ha llevado a cabo en un Centro Público en donde se presta atención psicológica de forma inmediata a las víctimas de agresiones sexuales.

\section{Instrumentos}

a) Variables sociodemográficas, circunstancias del proceso de victimización y apoyo familiar y social

La Entrevista Semiestructurada para Victimas de Agresiones Sexuales (Echeburúa et al., 1995) es un instrumento que eval- 
úa la historia de victimización sexual, la percepción de amenaza a la vida y las consecuencias jurídico-penales. Asimismo permite identificar la reacción del entorno familiar, los recursos de apoyo existentes, la victimización secundaria y el sentimiento de culpa. Por último, se reflejan las características sociodemográficas, los antecedentes psicopatológicos, la existencia de sucesos estresantes en el último año y el estado de salud de las víctimas.

\section{b) Variables psicopatológicas}

La Escala de Gravedad de Sintomas del Trastorno de Estrés Postraumático (EGS) (Echeburúa, Corral, Amor, Zubizarreta y Sarasua, 1997) es una escala heteroaplicada que evalúa la gravedad e intensidad de los síntomas de este trastorno según los criterios diagnósticos del DSM-IV-TR (APA, 2000) en víctimas de diferentes sucesos traumáticos. Esta escala está estructurada en un formato de tipo Likert, de 0 a 3, según la frecuencia e intensidad de los síntomas y consta de 17 ítems que corresponden a los criterios diagnósticos (5 hacen referencia a los síntomas de reexperimentación; 7 a los de evitación; y 5 a los de activación). El rango oscila de 0 a 51 en la escala global; de 0 a 15, en la subescala de reexperimentación; de 0 a 21, en la de evitación; y de 0 a 15, en la de activación.

Es una escala con una eficacia diagnóstica muy alta $(95.45 \%)$ si se establece un punto de corte global de 15 y unos puntos de corte parciales de 5 (con 2 puntos, al menos, en un síntoma), 6 (con 2 puntos, al menos, en tres síntomas) y 4 (con dos puntos, al menos, en dos síntomas) en la subescalas de reexperimentación, evitación y activación, respectivamente. Las propiedades psicométricas son muy satisfactorias (Echeburúa et al., 1997).

El Inventario de Ansiedad-Estado (STAI) (Spielberger, Gorsuch y Lushene, 1970) (versión española de TEA, 1982) es un autoinforme que consta de 20 ítems relacionados con la ansiedad-rasgo y de otros 20 relacionados con la ansiedadestado. El rango de puntuaciones oscila de 0 a 60 en cada escala. El punto de corte elegido para la población femenina en el STAI-Estado es de 31 (correspondiente al percentil 75). La fiabilidad test-retest es de .81 en la escala de ansiedad-rasgo, bastante más alta, como es lógico, que en la de ansiedad-estado $(r=.40)$. La consistencia interna varía de .83 a .92. Por otra parte, la validez convergente con otras medidas de ansiedad oscila entre .58 y .79 .

El Inventario de Depresión de Beck (BDI) (Beck, Rush, Shaw y Emery, 1979) (versión española de Vázquez y Sanz, 1997) es un autoinforme de 21 ítems (rango: 0-63 puntos) que mide la intensidad de los síntomas depresivos y da más importancia a los componentes cognitivos de la depresión que a los conductuales y somáticos. El punto de corte más utilizado para discriminar entre población sana y aquejada de sintomatología depresiva es de 18 (Beck, Brown y Steer, 1996). El coeficiente de fiabilidad por el método de las dos mitades es de .93. Desde la perspectiva de la validez convergente, la correlación con la evaluación clínica de la depresión oscila entre .62 y .66 .

La Escala de Valoración de la Depresión (HDRS) (Hamilton, 1960) (versión española de Conde y Franch, 1984) es un instrumento heteroaplicado que evalúa la sintomatología depresiva, especialmente en los componentes somáticos y conductuales. Se suele utilizar como medida complementaria al $B D I$. El punto de corte establecido es de 18 puntos. Las propiedades psicométricas son satisfactorias -tiene una fiabilidad entre observadores de .90 y el coeficiente de validez convergente es de .60- y, además, es muy sensible a los cambios terapéuticos.

El Cuestionario de Miedos Modificado (MFS-III) (Veronen y Kilpatrick, 1980), basado en el Cuestionario de Miedos de Wolpe y Lang (1964), cuenta con una subescala específica de 45 ítems de miedos referidos a la agresión sexual, que es la que se ha utilizado en esta investigación y que está estructurada en un formato de tipo Likert (de 1 a 5) en función del nivel de malestar que produce cada situación. El rango total de esta subescala es de 45 a 225. La fiabilidad test-retest es de 60 a .74. La consistencia interna oscila entre .81 y .94. Desde la perspectiva de la validez discriminante, este instrumento discrimina adecuadamente entre las víctimas y las no víctimas durante, al menos, 3 años después de la agresión sexual. La versión española de este cuestionario se puede encontrar en Echeburúa et al., 1995.

La Escala de Autoestima (EAE) (Rosenberg, 1965) tiene por objetivo evaluar el grado de satisfacción que tiene una persona consigo misma. Este autoinforme consta de 10 ítems generales que puntúan de 1 a 4 en una escala de tipo Likert (rango: 10-40 puntos). El punto de corte en la población adulta es de 29 , con una puntuación tanto mayor cuanto mayor es la autoestima. La fiabilidad test-retest es de $.85 \mathrm{y}$ el coeficiente alfa de consistencia interna es de .92. La validez convergente y la validez discriminante son asimismo satisfactorias. La versión española de esta escala se puede encontrar en Echeburúa y Corral (1998).

La Escala de Inadaptación (IG) (Echeburúa, Corral y Fernández-Montalvo, 2000) es un autoinforme que consta de 6 ítems (rango: 0-30) y mide el grado en que la situación de maltrato afecta a diferentes áreas de la vida cotidiana (trabajo, vida social, tiempo libre, relación de pareja o posibilidad de tenerla y relación familiar), así como también a nivel global. El punto de corte establecido es de 12 en la escala total y de 2 en cada uno de los ítems, con una puntuación tanto mayor cuanto mayor es la inadaptación. En esta investigación se ha utilizado el ítem que refleja el grado de inadaptación global a la vida cotidiana (rango: 0-5 puntos). Las propiedades psicométricas de fiabilidad y validez han resultado satisfactorias y están descritas en Echeburúa et al., 2000 .

\section{Modalidad terapéutica}

La modalidad terapéutica aplicada en esta investigación ha sido un tratamiento cognitivo-conductual en formato in- 
dividual que consta de 12 sesiones de una hora de duración con una periodicidad semanal y que tiene una duración aproximada de 3 meses.

Se trata de un programa que consta de 2 partes. La primera se centra en los aspectos motivacionales; y la segunda está más referida a las consecuencias psicopatológicas derivadas de los sucesos traumáticos vividos.

La intervención motivacional orientada a transmitir empatía y apoyo emocional a la víctima comprende las dos primeras sesiones. La segunda parte está dedicada específicamente al tratamiento de los síntomas psicopatológicos detectados, con tres niveles de intervención de 2,3 y 5 sesiones cada uno. Los componentes del programa inicial, con la distribución de sesiones correspondiente y su articulación en el conjunto del tratamiento, figuran descritos en otro lugar (Echeburúa et al., 1995) y están resumidos en la Tabla 1.

Tabla 1. Programa de tratamiento y diario de sesiones

MOTIVACIÓN (1 ${ }^{a}-2^{a}$ sesión)

a Aspectos motivacionales

- Percepción de seguridad en un marco terapéutico empático

- Apoyo emocional

\section{SÍNTOMAS PSICOPATOLÓGICOS}

\section{Primer nivel de intervención ( $3^{a}-4^{a}$ sesión)}

口 Catarsis emocional

Segundo nivel de intervención (5 $5^{a}-7^{a}$ sesión)

Intervención psicoeducativa

- Explicación del proceso de victimización y de las consecuencias psicológicas a corto y largo plazo

- Indicación de los aspectos positivos de su vida y de su persona

- Reevaluación cognitiva

- Distorsiones cognitivas (sentimientos de culpa, de ambivalencia e indefensión)

- Racionalización de la situación vivida

\section{Tercer nivel de intervención ( ${ }^{a}-12^{a}$ sesión)}

口 Entrenamiento en habilidades de afrontamiento

- Técnicas de distracción cognitiva

- Relajación muscular progresiva

- Técnicas de autoexposición

$>$ Recuperación de actividades cotidianas

$>$ Recuperación de actividades reforzantes

$>$ Normalización de la conducta sexual

Aunque el tratamiento psicológico tiene una estructura flexible, en función de la intensidad de los síntomas psicológicos detectados, cabe destacar que, excepto el último aspecto de la intervención del tercer nivel, se ha aplicado siempre en toda su extensión.

\section{Procedimiento}

El programa de evaluación y tratamiento descrito, elaborado conjuntamente por los cuatro firmantes del trabajo, se puso a prueba por primera vez en un estudio-piloto con 20 víctimas de agresiones sexuales aquejadas de un trastorno de estrés postraumático crónico (Echeburúa et al., 1997).

Las víctimas participantes en esta investigación fueron evaluadas en 2 sesiones. Asimismo se les explicó el contenido del programa y firmaron la hoja de consentimiento informado. En la primera sesión se obtuvieron los datos relacionados con las características biográficas y las circunstancias del proceso de victimización sexual y del apoyo familiar. En la segunda se evaluaron los síntomas psicopatológicos. Las siguientes evaluaciones se realizaron en el postratamiento y en los seguimientos de 1, 3, 6 y 12 meses.

La evaluación y el tratamiento se llevaron a cabo por un equipo de psicólogas clínicas con formación en violencia de género y con, al menos, tres años de experiencia clínica en terapia cognitivo-conductual. Además, se realizó una supervisión por las directoras del programa, que cuentan con una dilatada experiencia en el tratamiento de las víctimas de agresiones sexuales.

\section{Análisis de datos}

Los análisis estadísticos se realizaron con el programa SPSS y se utilizó la versión 15.0 para Windows. Para describir las características de la muestra (las variables biográficas e historia de victimización y las variables psicopatológicas) se ha llevado a cabo un análisis de carácter descriptivo (medias, desviaciones típicas y porcentajes). Y para analizar los resultados del tratamiento se utilizó un análisis de frecuencias y de comparación de medias y se recurrió como prueba de contraste a un análisis de varianza de un factor con medidas repetidas (valores de $F$ y $t$ ).

\section{Resultados}

En este apartado se incluyen las características y las variables psicopatológicas de la muestra, así como los resultados del tratamiento.

\section{Características de la muestra}

A continuación se describen las circunstancias del proceso de victimización y las variables más significativas relacionadas con la víctima y con el apoyo familiar de las personas seleccionadas en el estudio $(n=131)$.

Respecto a la historia de victimización sexual, la inmensa mayoría sufrió abusos repetidos $(n=120 ; 91.6 \%)$ por parte de un familiar $(n=108 ; 82.4 \%)$ durante, al menos, más de un año, excepto en unos pocos casos en que hubo un solo episodio $(n=11 ; 8.4 \%)$. El intervalo de edad más frecuente en el que se produjo el último episodio oscila entre los 9 y 13 años $(n=61 ; 46.6 \%)$, siendo la media de edad de 12.7 años (rango: 5-20 años). Por lo que al tipo de agresión se refiere, el abuso sexual con penetración se consumó en casi la mitad de las víctimas $(n=53 ; 40.5 \%)$. Sin embargo, la mayoría no sufrió lesiones $(n=122 ; 93.1 \%)$ ni tuvo percepción de amenaza a la vida $(n=117 ; 89.3 \%)$. 
En cuanto a las variables más significativas relacionadas con la víctima y con el apoyo familiar, la mayoría no tenía otra historia de victimización $(n=88 ; 67.2 \%)$ ni problemas de consumo abusivo de alcohol $\mathrm{u}$ otras drogas $(n=120 ; 91.6 \%)$, pero más de la mitad de las víctimas estuvieron expuestas a sucesos estresantes en el último año $(n=66 ; 50.4 \%)$. Por otro lado, la mayor parte de ellas disponían de una red de apoyo social $(n=82 ; 62.6 \%)$.

Las víctimas revelaron mayoritariamente los sucesos a la familia $(n=109 ; 83.2 \%)$, pero casi la mitad no se sintieron creídas $(n=44 ; 40.4 \%)$ ni recibieron un apoyo adecuado dentro del contexto familiar $(n=74 ; 67.9 \%)$ ni tampoco denunciaron los abusos sexuales $(n=114 ; 87 \%)$.

Por tanto, no es de extrañar que el tiempo medio transcurrido desde la última agresión hasta el momento de iniciar el proceso terapéutico haya sido de 15 años, aunque el rango era amplio y oscilaba entre las 2 semanas y los 44 años.

\section{Síntomas psicopatológicos}

Las variables psicopatológicas medidas en la evaluación pretratamiento, que abarcó a 121 víctimas (10 no se incorporaron al tratamiento), son el trastorno de estrés postraumático y el malestar emocional (sintomatología ansiosodepresiva, inadaptación a la vida cotidiana y conductas de evitación sexual).

Las víctimas de abuso sexual en la infancia padecían un trastorno de estrés postraumático en el 44.6\% $(n=54)$ de los casos evaluados y, desde una perspectiva dimensional, presentaron un alto nivel de gravedad, con una puntuación global $(X=29.81 ; D T=7.00)$ claramente por encima del punto de corte establecido. A efectos de comprobar la fiabilidad entre observadores del diagnóstico del TEPT con la EGS, se calculó el coeficiente kappa, que fue más bien alto (.91).

En cuanto al malestar emocional, el nivel de ansiedad era muy alto (STAI-E $X=37.17 ; D T=11.20$ ), con bastantes miedos asociados al abuso sexual (MFS III $X=110.43$; $D T=31.61)$ y con temores perturbadores relacionados con estímulos asociados directamente a la experiencia de la agresión y a indicadores de vulnerabilidad (tabla 2). Además, las víctimas que tenían pareja en el momento de la evaluación psicopatológica $(n=47 ; 38.8 \%)$ evitaban mantener relaciones sexuales en el $44.7 \%(n=21)$ de los casos.

Tabla 2. Miedos a largo plazo $(\mathrm{n}=121)$.

\begin{tabular}{lcc}
\hline Estímulos asociados con el abuso sexual & $N$ & $\boldsymbol{\%}$ \\
\hline Coito anal & 62 & 51.23 \\
Sensación de no poder respirar & 54 & 44.62 \\
Escenas violentas en la TV o en el cine & 50 & 41.32 \\
Acto sexual & 49 & 40.49 \\
Gritos & 49 & 40.49 \\
\hline Estímulos indicadores de vulnerabilidad & & \\
\hline Andar por una calle poco iluminada & 62 & 51.23 \\
Sentir que no creen lo que digo & 59 & 48.76 \\
Notar gente que me sigue o va detrás de mí & 54 & 44.62 \\
Oír ruidos inesperados & 49 & 40.49 \\
\hline
\end{tabular}

Asimismo las víctimas mostraban una sintomatología depresiva moderada tanto en los componente cognitivos (BDI $X=21.86 ; D T=10.71)$ como conductuales y psicofisiológicos (HDRS $X=24.62 ; D T=12.14$ ), con sentimientos de culpa por los abusos sufridos en la mayoría de los casos $(n=68 ; 56.2 \%)$, con baja autoestima (EAE $X=25.85$; $D T=5.96)$ y con dificultades globales de adaptación a la vida cotidiana $(I G X=3.5 ; D T=1.31)$.

Por último, el $66.9 \%(n=81)$ de las víctimas presentaron un grado de malestar clínicamente significativo cuando se valoraba conjuntamente el nivel de ansiedad, de depresión e inadaptación, atendiendo a los puntos de corte de los instrumentos utilizados ( $\geq 24$ en el STAI-E; $\geq 18$ en el BDI; y $\geq 3$ en la $I G)$.

\section{Resultados del tratamiento}

En relación con los rechazos y abandonos, hubo 10 pacientes (el 7.6\%) que rechazaron el tratamiento propuesto, después de habérselo explicado. De las víctimas que iniciaron el programa de intervención $(n=121)$, hubo 87 (el $71.9 \%$ ) que lo completaron y 34 (el 28.1\%) que lo abandonaron prematuramente.

Respecto al éxito con el tratamiento, en este estudio se ha utilizado un indicador múltiple de éxito terapéutico: desaparición del TEPT (puntuación $<15$ en la EGS), superación del malestar emocional clínicamente significativo (según los puntos de corte de las siguientes medidas: puntuación $<24$ en el STAI-E, $<18$ en el BDI y $<3$ en la $I G$ ) y normalización de la conducta sexual.

La tasa de éxitos en la evaluación postratamiento en relación con el TEPT es del 90.7\% (es decir, de las 43 víctimas que sufrían TEPT al comienzo de la terapia 39 lo superaron). Si bien hay una pérdida de 11 sujetos (el 25.58\% sobre el total de los casos tratados con TEPT) en los diversos controles de seguimiento, la tasa de éxitos se mantiene relativamente constante en los diferentes momentos de la evaluación y llega hasta un $74.42 \%(n=32)$ en el último seguimiento de los 12 meses (Tabla 3).

Tabla 3. Tasa de éxitos en relación con la desaparición del TEPT $(n=43)^{*}$. EVALUACIÓN $(N=43) \quad$ ÉXITOS FRACASOS

\begin{tabular}{|c|c|c|}
\hline - & $N(\%)$ & $N(\%)$ \\
\hline POSTRATAMIENTO & $39(90.70)$ & $4(9.30)$ \\
\hline EVALUACIÓN DEL & ÉXITOS & Pérdidas en el \\
\hline SEGUIMIENTO & $N(\%)$ & $\begin{array}{l}\text { seguimiento } \\
N(\%)\end{array}$ \\
\hline $1 \mathrm{MES}$ & $38(88.37)$ & $5(11.62)$ \\
\hline 3 MESES & 35 (81.39) & $3(6.98)$ \\
\hline $6 \mathrm{MESES}$ & $34(79.06)$ & $1(2.32)$ \\
\hline 12 MESES & $32(74.42)$ & $2(4.65)$ \\
\hline
\end{tabular}

A su vez, la tasa de éxitos en la evaluación postratamiento respecto al malestar emocional es del $64.52 \%$ (es decir, de 
las 62 víctimas que sufrían malestar emocional al comienzo de la terapia 40 lo superaron). Estos resultados se consolidan e incluso aumentan ligeramente en los distintos controles de seguimiento (una tasa del $70.97 \%$ a los 12 meses), a pesar de los 15 casos (el $24.19 \%$ ) perdidos en los seguimientos (Tabla 4).

Tabla 4. Tasa de éxitos en relación con la desaparición del malestar emocional $(n=62)^{*}$.

\begin{tabular}{|c|c|c|c|}
\hline $\begin{array}{l}\text { EVALUACIÓN } \\
(N=62)\end{array}$ & $\begin{array}{l}\text { EXITOS } \\
N(\%)\end{array}$ & $\begin{array}{l}\text { FRACASOS } \\
N(\%)\end{array}$ & \\
\hline POSTRATAMIENTO & $40(64.52)$ & $22(35.48)$ & \\
\hline $\begin{array}{l}\text { EVALUACIÓN DEL } \\
\text { SEGUIMIENTO }\end{array}$ & $\begin{array}{l}\text { ÉXITOS } \\
N(\%)\end{array}$ & FRACASOS & $\begin{array}{l}\text { Pérdidas } \\
\text { en el } \\
\text { eguimiento }\end{array}$ \\
\hline & & $N(\%)$ & $N(\%)$ \\
\hline $1 \mathrm{MES}$ & $45(72.58)$ & $10(16.12)$ & $7(11.29)$ \\
\hline 3 MESES & 45 (81.39) & $6(9.68)$ & $4(6.45)$ \\
\hline 6 MESES & $43(69.35)$ & $5(8.06)$ & $3(4.84)$ \\
\hline 12 MESES & $44(70.97)$ & $3(4.84)$ & $1(1.61)$ \\
\hline
\end{tabular}

* La muestra inicial de víctimas con malestar emocional fue de 81 mujeres (el $66.9 \%$ de la muestra total). Sin embargo, 19 de ellas (el 23.4\%) no completaron el tratamiento.

Por último, en cuanto a la normalización de la conducta sexual, la tasa de éxitos ha sido alta, tanto en la evaluación postratamiento $(n=14 ; 87.50 \%)$ como en los diversos controles de seguimiento ( $n=12,75 \%$, a los 12 meses). El tamaño de la muestra en esta variable ha sido reducido $(n=16)$ porque muchas de las víctimas o no tenían pareja $(n=74$; $61.1 \%$ ) o no presentaban este problema en la evaluación pretratamiento $(n=26 ; 55.3 \%)$ (Tabla 5).

Tabla 5. Tasa de éxitos en relación con la normalización de la conducta sexual $(n=16)^{*}$.

\begin{tabular}{|c|c|c|c|}
\hline $\begin{array}{l}\text { EVALUACIÓN } \\
(N=16)\end{array}$ & $\begin{array}{l}\text { EXITOS } \\
N(\%)\end{array}$ & $\begin{array}{l}\text { FRACASOS } \\
N(\%)\end{array}$ & \\
\hline POSTRATAMIENTO & $13(81.25)$ & $3(18.75)$ & \\
\hline $\begin{array}{l}\text { EVALUACIÓN DEL } \\
\text { SEGUIMIENTO }\end{array}$ & $\begin{array}{l}\text { ÉXITOS } \\
N(\%)\end{array}$ & FRACASOS & $\begin{array}{l}\text { Pérdidas } \\
\text { en el } \\
\text { guimiento }\end{array}$ \\
\hline & & $N(\%)$ & $N(\%)$ \\
\hline $1 \mathrm{MES}$ & $14(87.50)$ & $1(6.25)$ & $1 \quad(6.25)$ \\
\hline 3 MESES & $14(87.50)$ & $1(6.25)$ & \\
\hline 6 MESES & $13(81.25)$ & $1(6.25)$ & $1 \quad(6.25)$ \\
\hline 12 MESES & $12(75.00)$ & $1(6.25)$ & $1 \quad(6.25)$ \\
\hline
\end{tabular}

Desde una perspectiva dimensional, en la tabla 6 se muestran las medias y las desviaciones típicas de todas las medidas utilizadas en las diversas evaluaciones, así como los valores de $F$ y $t$ en el Anova de medidas repetidas. En todas las variables estudiadas se pone de manifiesto una gran mejoría entre las evaluaciones pre y postratamiento, que se mantiene constante e incluso aumenta hasta el seguimiento de los 12 meses.

Tabla 6. Medias y desviaciones típicas de las variables psicopatológicas en las evaluaciones. Valores de $F$ y $t$ en el análisis de varianza de medidas repetidas $(n=121)$.

\begin{tabular}{|c|c|c|c|c|}
\hline Variables & $X$ & $(D T)$ & Evaluaciones & $\mathrm{F} / \mathrm{t}$ \\
\hline INTENSIDAD DEL TEPT & & & & $F=283.404 * * *$ \\
\hline$(E G S)(0-51)$ & & & & $t$ \\
\hline Pretratamiento & 20.18 & $(11.21)$ & Pre.-Post & $13.465 * * * \quad(+++)$ \\
\hline Postratamiento & 8.44 & $(6.07)$ & Pre.-12 meses & $15.338 * * * \quad(+++)$ \\
\hline $1 \mathrm{mes}$ & 5.76 & $(4.25)$ & Post.-12 meses & $7.939 * * * \quad(+++)$ \\
\hline 3 meses & 4.63 & $(3.36)$ & & \\
\hline 6 meses & 4.28 & $(3.45)$ & & \\
\hline 12 meses & 3.90 & (3.11) & & \\
\hline ANSIEDAD (STAI-E) & & & & $F=558.238 * * *$ \\
\hline$(0-60)$ & & & & $t$ \\
\hline Pretratamiento & 37.17 & $(11.20)$ & Pre.-Post & $18.849 * * * \quad(+++)$ \\
\hline Postratamiento & 18.27 & $(8.93)$ & Pre.-12 meses & $21.519 * * * \quad(+++)$ \\
\hline $1 \mathrm{mes}$ & 14.51 & $(7.07)$ & Post.-12 meses & $6.447 * * * \quad(+++)$ \\
\hline 3 meses & 13.00 & $(7.30)$ & & \\
\hline 6 meses & 12.04 & $(7.08)$ & & \\
\hline 12 meses & 11.86 & $(6.48)$ & & \\
\hline DEPRESIÓN (BDI) & & & & $F=283.370$ *** \\
\hline$(0-63)$ & & & & $t$ \\
\hline Pretratamiento & 21.86 & $(10.71)$ & Pre.-Post & $13.916 * * *(+++)$ \\
\hline Postratamiento & 9.02 & $(7.48)$ & Pre.-12 meses & $15.915 * * *(+++)$ \\
\hline $1 \mathrm{mes}$ & 6.87 & $(5.37)$ & Post.-12 meses & $3.982 * * *(+++)$ \\
\hline 3 meses & 6.42 & $(5.47)$ & & \\
\hline 6 meses & 6.22 & $(4.92)$ & & \\
\hline 12 meses & 6.18 & $(4.54)$ & & \\
\hline DEPRESIÓN (HDRS) & & & & $F=321.658 * * *$ \\
\hline$(0-62)$ & & & & $t$ \\
\hline Pretratamiento & 24.62 & $(12.14)$ & Pre.-Post & $12.773 * * *(+++)$ \\
\hline Postratamiento & 12.22 & $(7.46)$ & Pre. -12 meses & $14.812 * * *(+++)$ \\
\hline $1 \mathrm{mes}$ & 8.83 & $(5.98)$ & Post.-12 meses & $7.560 * * *(+++)$ \\
\hline 3 meses & 7.47 & $(5.56)$ & & \\
\hline
\end{tabular}




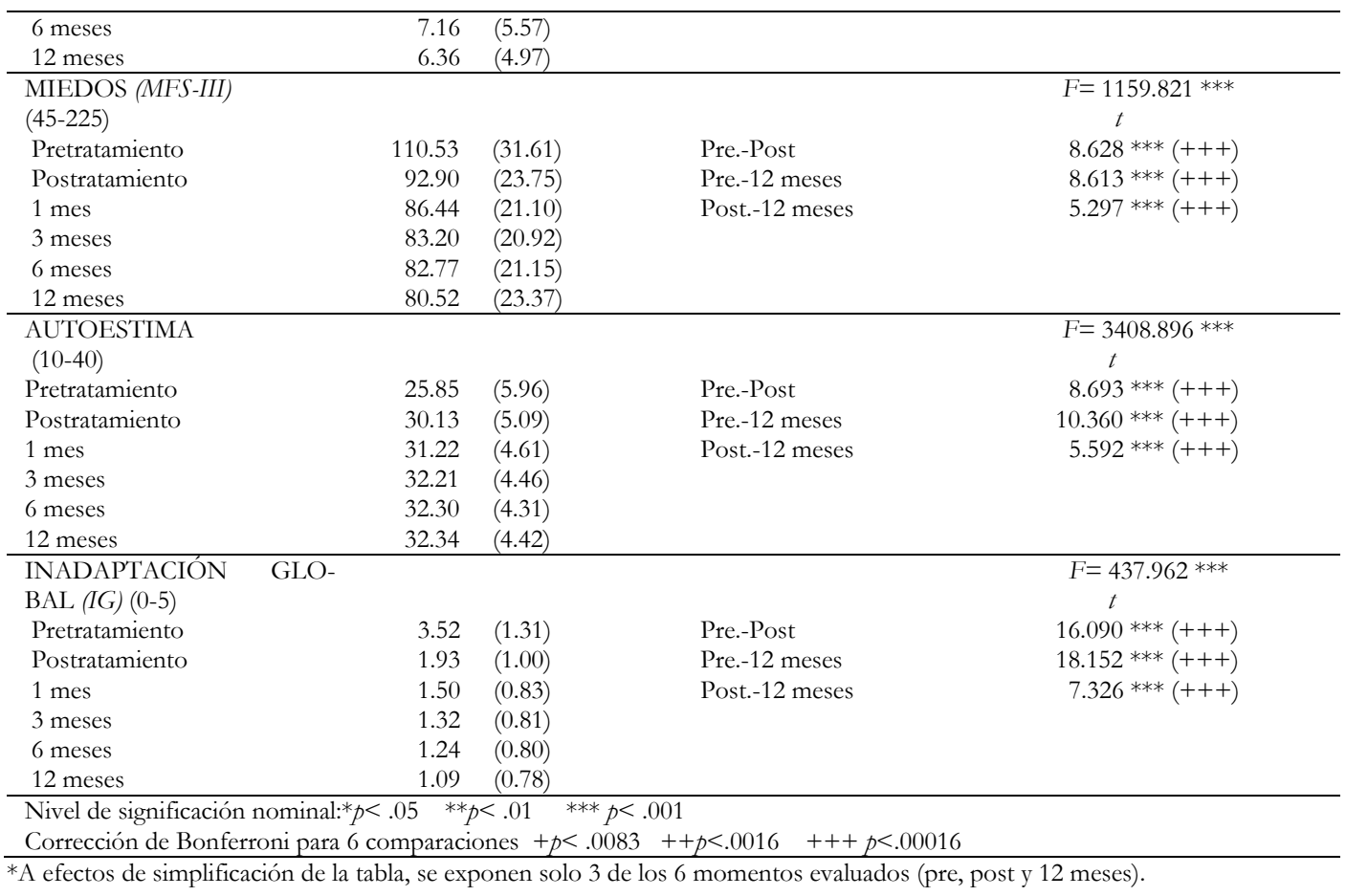

\section{Discusión y conclusiones}

En esta investigación se ha estudiado a 131 víctimas adultas de abuso sexual en la infancia que han buscado ayuda terapéutica más de 15 años después de haber sufrido unos abusos sexuales graves y repetidos a manos de un familiar. Se trata, por tanto, de una muestra amplia y representativa de mujeres jóvenes (en torno a 25-30 años) que presentaban un malestar emocional crónico que desbordaba sus recursos psicológicos de afrontamiento y que interfería negativamente en su vida cotidiana, tal y como se muestra también en otros trabajos (por ejemplo, Cortés et al., 2011; Fergusson et al., 2008). La búsqueda tardía de tratamiento se debe a la cronicidad de los síntomas, a los problemas afectivos y sexuales en las relaciones de pareja o a los cambios adaptativos frecuentes en personas en esta fase evolutiva de la vida (Echeburúa et al., 1997).

Por lo que se refiere a las características psicopatológicas de la muestra, las víctimas presentaban cuadros clínicos diversos porque el abuso sexual es un suceso traumático, no un diagnóstico clínico (Maniglio, 2009). Los trastornos más frecuentes en la muestra estudiada eran el malestar emocional (un $66.9 \%$ del total), el trastorno de estrés postraumático (un $44.6 \%$ del total) y las conductas de evitación sexual (un $44.7 \%$ del total). Los cuadros clínicos podían ser razonablemente atribuidos a los efectos del abuso sexual porque en dos de cada tres casos no presentaban otra vía de victimización ni las víctimas eran consumidoras abusivas de alcohol o drogas. En las víctimas había dos factores de agravamiento de los síntomas: casi la mitad de ellas sufrieron un abuso sexual con penetración y también casi la mitad no se sintie- ron creídas por la familia cuando revelaron la existencia de los abusos.

El programa de tratamiento expuesto se ha llevado a cabo en un centro especializado en un medio comunitario, en donde las víctimas han acudido al programa por iniciativa propia, por los Servicios Sociales o por derivación de los Centros de Salud Mental. La intervención propuesta, de 12 sesiones, ha sido estructurada en diversos módulos (aspectos motivacionales y de empatía, psicoeducación, reevaluación cognitiva y habilidades de afrontamiento), se ha aplicado en un formato individual a una muestra más bien grande (131 mujeres) y se ha llevado a cabo un seguimiento suficientemente amplio (1 año, con controles periódicos). El tratamiento propuesto en esta investigación, tanto en el enfoque teórico como en el formato y la duración propuestos, es coherente con las recientes revisiones meta-analíticas de Peleikis y Dahl (2005) y de Taylor y Harvey (2010).

A diferencia de otros estudios previos centrados exclusivamente en el trastorno de estrés postraumático (Echeburúa et al., 1997; Foa y Rothbaum, 1998; Resnick y Newton, 1992; Smucker et al., 1995), el objetivo de esta investigación ha sido la mejoría terapéutica de las víctimas en un espectro de conductas más amplio (trastorno de estrés postraumático, malestar emocional y conductas de evitación sexual), tal como se ha manifestado también en otros estudios (Martsolf y Draucker, 2005; Pearson, 1994; Peleikis y Dahl, 2005; y Taylor y Harvey, 2010).

Respecto al balance de la terapia, cabe señalar, en primer lugar, que ha habido una tasa de rechazos $(7.6 \%)$ y de abandonos $(28.1 \%)$ del programa relativamente alta. Al margen de ciertas variables coyunturales, como enfermedades o 
cambios de residencia, una circunstancia explicativa de este hecho puede ser la dureza de una terapia que obliga a la víctima a confrontar cogniciones y emociones negativas (vergüenza, culpa, odio o repugnancia) que, además, están relacionadas con personas de su propia familia.

En las víctimas que han completado el tratamiento $(71.9 \%)$ los resultados obtenidos han sido muy positivos, si bien variables en los distintos objetivos terapéuticos. Es decir, en el postratamiento ha habido una desaparición del trastorno de estrés postraumático y de las conductas de evitación sexual en el $90.7 \%$ y el $81.2 \%$ de los casos, respectivamente, pero la desaparición del malestar emocional ha sido algo más reducida (64.5\%), quizá porque esta última variable es más resistente al cambio. Un aspecto destacable es que las tasas de éxito terapéutico se mantienen relativamente constantes (e incluso aumentan en el caso del malestar emocional) hasta el seguimiento de los 12 meses. Esta respuesta favorable al tratamiento ha aparecido también en las revisiones de Peleikis y Dahl (2005) y de Taylor y Harvey (2010).

Los resultados encontrados en este estudio son muy positivos si se tiene en cuenta que las víctimas presentaban un cuadro clínico severo y crónico ligado a un abuso sexual sufrido hacía más de 15 años, que el programa de tratamiento ofertado fue breve (12 sesiones con una periodicidad semanal) y que el éxito terapéutico obtenido se mantuvo en el seguimiento de 1 año. Este último dato es meritorio porque en la mayor parte de los estudios realizados no se han controlado los efectos del tratamiento más allá de los 6 meses (Taylor y Harvey, 2010). Se trata, por tanto, de un estudio que ha

\section{Referencias}

Beck, A.T., Brown, G.K. y Steer, R.A. (1996). Beck Depression Inventory (BDIII). San Antonio, TX: The Psychological Corporation.

Beck, A.T., Rush, A.J., Shaw, B.F. y Emery, G. (1979). Cognitive therapy of depression. New York: Guilford Press.

Briere, J. y Elliott, D.M. (2003). Prevalence and psychological sequelae of self-reported childhood physical and sexual abuse in a general population sample of men and women. Child Abuse and Neglect, 27, 1205-1222.

Conde, V. y Franch, J.I. (1984). Escalas de evaluación comportamental para la cuantificación de la sintomatología psicopatológica en los trastornos angustiosos y depresivos. Madrid: Laboratorios Upjohn.

Cortés, M.R., Cantón-Cortés, D. y Cantón, J. (2011). Consecuencias a largo plazo del abuso sexual infantil: papel de la naturaleza y continuidad del abuso y del ambiente familiar. Behavioral Psychology/Psicologia Conductual, 19, 41-56.

Echeburúa, E. y Corral, P. (1998). Manual de violencia familiar. Madrid. Siglo XXI.

Echeburúa, E. y Corral, P. (2007). Intervención en crisis en víctimas de sucesos traumáticos: ¿cuándo, cómo y para qué? Psicología Conductual, 15, 373-387.

Echeburúa, E. y Guerricaechevarría, C. (2011). Tratamiento psicológico de las víctimas de abuso sexual infantil intrafamiliar: un enfoque integrador. Behavioral Psychology/Psicologia Conductual, 19, 469-486.

Echeburúa, E. y Subijana, I.J. (2008). Guía de buena práctica psicológica en el tratamiento judicial de los niños abusados sexualmente. International Journal of Clinical and Health Psychology, 8, 733-749.

Echeburúa, E., Corral, P. y Amor, P.J. (2002). Evaluación del daño psicológico en las víctimas de delitos violentos. Psicothema, 14 (supl.), 139-146. mostrado la existencia de un tratamiento efectivo, pero también eficiente porque se puede implementar en la práctica habitual de los Centros de Atención a la Víctima sin necesidad de grandes inversiones económicas o en recursos humanos (Echeburúa y Corral, 2007; Echeburúa, Corral, Sarasua y Zubizarreta, 1996).

Algunas limitaciones de este estudio son que carece de un grupo de control (difícil de obtener en este tipo de investigaciones) y que, al tratarse de un programa con muchos componentes, resulta difícil establecer el peso específico de cada uno de ellos. Además de depurar el contenido del tratamiento, resultaría de interés en el futuro conocer el perfil de las víctimas que completan el tratamiento en relación con el de las que no lo hacen o lo rechazan, así como poner a prueba distintos formatos de tratamiento (individual/grupal) (Echeburúa, Sarasua, Zubizarreta y Corral, 2009; Sarasua, Zubizarreta, Corral y Echeburúa, 2012). Una limitación adicional es que en este estudio, a efectos de homogeneizar la muestra, se han excluido víctimas con trastornos mentales graves (trastorno bipolar y límite, fundamentalmente) y que, por tanto, las conclusiones acerca de la eficacia de la terapia no se pueden generalizar a este tipo de víctimas. Parece de interés abordar este tema en estudios futuros. Por último, un reto prioritario es diseñar estrategias motivacionales para el tratamiento, a efectos de reducir la tasa de rechazos y abandonos, y adaptar el programa de tratamiento a las necesidades específicas de cada víctima (Echeburúa, FernándezMontalvo y Corral, 2008; Echeburúa, Sarasua, Zubizarreta, Amor y Corral, 2010).
Echeburúa, E., Corral, P. y Fernández-Montalvo, J. (2000). Escala de Inadaptación: propiedades psicométricas en contextos clínicos. Análisis y Modificación de Conducta, 26, 325-340.

Echeburúa, E., Fernández-Montalvo, J. y Corral, P. (2008). ¿Hay diferencias entre la violencia grave y la violencia menos grave contra la pareja?: un análisis comparativo. International Journal of Clinical and Health Psychology, 8, 355-382.

Echeburúa, E., Corral, P., Amor, P.J., Zubizarreta, I. y Sarasua, B. (1997). Escala de gravedad de síntomas del trastorno de estrés postraumático: Propiedades psicométricas. Análisis y Modificación de Conducta, 23, 503526.

Echeburúa, E., Corral, P., Sarasua, B. y Zubizarreta, I. (1996). Treatment of acute posttraumatic stress disorder in rape victims: An experimental study. Journal of Anxiety Disorders, 10, 185-199.

Echeburúa, E., Corral, P., Zubizarreta, I. y Sarasua, B. (1995). Trastorno de estrés postraumático crónico en víctimas de agresiones sexuales. La Coruña: Fundación Paideia.

Echeburúa, E., Corral, P., Zubizarreta, I. y Sarasua, B. (1997). Psychological treatment of chronic posttraumatic stress disorder in victims of sexual aggression. Behavior Modification, 21, 433-456.

Echeburúa, E., Sarasua, B., Zubizarreta, I. y Corral, P. (2009). Evaluación de la eficacia de un tratamiento cognitivo-conductual para hombres violentos contra la pareja en un marco comunitario: una experiencia de 10 años (1997-2007). International Journal of Clinical and Health Psychology, 9, 199-217.

Echeburúa, E., Sarasua, B., Zubizarreta, I., Amor, P.J. y Corral, P. (2010). Variables predictoras del rechazo, abandono y fracaso terapéutico en hombres violentos contra su pareja tratados psicológicamente en un marco comunitario. International Journal of Clinical and Health Psychology, 10, 403-420. 
Fergusson, D.M., Boden, J.M. y Horwood, L.J. (2008). Exposure to childhood sexual and physical abuse and adjustment in early adulthood. Child Abuse and Neglect, 32, 607-619.

Flitter, J.M.K., Elhai, J.D. y Gold, S.N. (2003). MMPI-2 F scale elevations in adult victims of child sexual abuse. Journal of Traumatic Stress, 16, 269274.

Foa, E.B. y Rothbaum, B.O. (1998). Treating the trauma of rape: Cognitivebehavioral therapy for PTSD. New York: Guilford Press.

Foa, E.B., Keane, T.M. y Friedman, M.J. (2003). Tratamiento del estrés postraumático. Barcelona: Ariel.

Hamilton, M. (1960). A rating scale for depression. Journal of Neurology, Neurosurgery and Psychiatry, 23, 53-65.

James, R.K. (2008). Crisis intervention strategies (6 ${ }^{\text {th }}$ edition), Thomson: Belmont, CA.

Jehu, D. (1989). Mood disturbances among women clients sexually abused in childhood. Journal of Interpersonal Violence, 4, 164-184.

Katz, R.C. y Watkins, P.L. (1998). Adult victims of child sexual abuse. En: A.S. BellacK y M. Hersen (Eds.), Comprehensive Clinical Psychology (vol. 9, pags. 291-306). Elsevier: Amsterdam.

Kendler, K.S., Bulik, C.M., Silberg, J., Hettema, J.M., Myers, J. y Prescott, C.A. (2000). Childhood sexual abuse and adult psychiatric and substance use disorders in women: An epidemiological and cotwin control analysis. Archives of General Psychiatry, 57, 953-959.

Kilpatrick, D.G. y Calhoum, K.S. (1988). Early behavioral treatment for rape trauma: efficacy or artifact? Behavior Therapy, 19, 421-427.

Maniglio, R. (2009). The impact of child sexual abuse on health: A systematic review of reviews. Clinical Psychology Review, 29, 647-657.

Martsolf, D.F. y Draucker, C.B. (2005). Psychotherapy approaches for adult survivors of childhood sexual abuse: An integrative review of outcomes research. Issues in Mental Health Nursing, 26, 801-825.

McLean, L.M. y Gallop, R. (2003). Implications of childhood sexual abuse for adult borderline personality disorder and complex posttraumatic stress disorder. American Journal of Psychiatry, 160, 369-371.

Patró, R., Corbalán, F. y Limiñana, R. (2008). Depresión en mujeres maltratadas: relaciones con estilos de personalidad, variables contextuales y de la situación de violencia. Anales de Psicología, 23, 118-124.

Pearson, Q. (1994). Treatment techniques for adult female survivors of childhood sexual abuse. Journal of Counseling and Development, 73, 32-37.
Peleikis, D.E. y Dahl, A.A. (2005). A systematic review of empirical studies of psychotherapy with women who were sexually abused as children. Psychotherapy Research, 15, 304-315.

Pereda, N., Gallardo-Pujol y Jiménez Padilla, R. (2011). Trastornos de personalidad en víctimas de abuso sexual infantil. Actas Españolas de Psiquiatría, 39, 131-139.

Pereda, N., Guilera, G., Forns, M y Gómez-Benito, J. (2009). The prevalence of child sexual abuse in community and student samples: A metaanalysis. Clinical Psychology Review, 29, 328-338.

Putnam, F. (2003). Ten-year research update review: Child sexual abuse. Journal of the American Academy of Child and Adolescent Psychiatry, 42, 269-278.

Resnick, H.S. y Newton, T. (1992). Assessment and treatment of post-traumatic stress disorder in adult survivors of sexual assault. En: D.W. Foy (Ed.), Treating PTSD: Cognitive-behavioral strategies (pags. 99-126). New York: Guilford.

Rosenberg, M. (1965). Society and the Adolescent Self Image. Princeton: Princeton University Press.

Sarasua, B., Zubizarreta, I., Corral, P. y Echeburúa, E. (2012). Factores de vulnerabilidad y de protección del impacto emocional en mujeres adultas víctimas de agresiones sexuales. Terapia Psicológica, 30, 5-16.

Smucker, M., Dancu, C., Foa, E.B. y Niederee, J. (1995). Imagery rescripting. A new treatment for survivors of childhood sexual abuse from post traumatic stress. Journal of Cognitive Psychotherapy, 9, 3-17.

Spielberger, C.D., Gorsuch, R.L. y Lushene, R.E. (1970). Manual of State/Trait Anxiety Inventory. Palo Alto CA: Consulting Psychologists Press (versión española, TEA, 1982).

Taylor, J.E. y Harvey, S.T. (2010). A meta-analysis of the effects of psychotherapy with adults sexually abused in childhood. Clinical Psychology Review, 30, 749-767.

Vázquez, C. y Sanz, J. (1997). Fiabilidad y valores normativos de la versión española del Inventario para la Depresión de Beck de 1978/Reliability and norm data of the Spanish versión of the 1978 Beck Depression Inventory. Clínica y Salud, 8, 403-422.

Veronen, L. J. y Kilpatrick, D.G. (1980). Self reported fears of rape victims: a preliminary investigation. Behavior Modification, 4, 383-396.

Wolpe, J. y Lang, P.L. (1964). A fear survey for use in behavior therapy. Behaviour Research and Therapy, 2, 27-30.

(Articulo recibido: 25-1-2012, revisado: 22-3-2012, aceptado: 23-3-2012) 